\title{
ON THE INERTIA GROUPS OF FIBRE BUNDLES
}

\author{
MICHAEL FRAME
}

\begin{abstract}
A subgroup $\tilde{I}\left(M \times S^{i}\right)$ of the inertia group $I\left(M \times S^{i}\right)$ is defined and shown to lie in $I(C)$ for every fibre bundle $M^{n} \rightarrow C \rightarrow N^{i}$. For certain $M$, examples of nontrivial elements in $\tilde{I}\left(M \times S^{i}\right)$ are constructed using the $\tau$ pairing of Milnor-Munkres-Novikov. For compact mapping tori $M_{g}$ it is shown that $I\left(M_{g}\right)=I\left(M \times S^{1}\right)$ if $\pi_{1} M$ is finite and $\mathrm{Wh}\left(\pi_{1} M\right)=0$.
\end{abstract}

1. Introduction. Recall that an exotic $n$-sphere is a smooth $n$-manifold homeomorphic to, but not necessarily diffeomorphic to, $S^{n}$. Under connected sum the exotic $n$-spheres form a group, denoted $\theta_{n}$. Letting $\Gamma^{n}=\pi_{0} \operatorname{Diff}\left(S^{n-1}\right)$, the map $\Gamma^{n} \rightarrow$ $\theta_{n}$ given by $[f] \rightarrow D^{n} \cup_{f} D^{n}=\Sigma(f)$ is an isomorphism for $n \geq 5$ [13]. The inertia group of a smooth $n$-manifold $M$ is defined by $I(M)=\left\{\Sigma \in \theta^{n}: \Sigma \# M \simeq\right.$ $M\}$, and it is a consequence of the disc theorem of Cerf and Palais that $\Sigma(f) \in$ $I(M)$ if and only if there is a diffeomorphism $F: M \backslash \operatorname{int} D^{n} \rightarrow M \backslash \operatorname{int} D^{n}$ satisfying $F \mid \partial D^{n}=f$. In this way it is seen that $I(M)$ measures the stability of the differentiable structure of $M$ under perturbations of an $n$-disc.

Many computations of inertia groups have been performed $[6,9,10,12,15,17$ among others]. In this paper a subgroup $\tilde{I}\left(M \times S^{i}\right)$ of $I\left(M \times S^{i}\right)$ is shown to lie in $I(C)$ for every fibre bundle $M^{n} \rightarrow C \rightarrow N^{i}$. Examples are constructed for which $\tilde{I}\left(M \times S^{i}\right) \neq 0$, and in particular for $N=S^{1}$ it is shown that $I\left(M \times S^{1}\right)=I(C)$ for every $C$ if $\pi_{1} M$ is finite and satisfies $\mathrm{Wh}\left(\pi_{1} M\right)=0$.

2. A special inertia group. Fix a point $* \in S^{i}$ and define $\tilde{I}\left(M^{n} \times S^{i}\right)=$ $\left\{\Sigma \in \theta_{n+i}:\right.$ there is a diffeomorphism $G:\left(M \times S^{i}\right) \# \Sigma \rightarrow M \times S^{i}$ with $G \mid M \times$ $\{*\}=$ identity $\}$. Of course, it suffices that $G(M \times\{*\})=M \times\{*\}$ since then $((G \mid M \times\{*\}) \times \text { id })^{-1} \circ G$ restricts to the identity on $M \times\{*\}$. Alone, this special inertia group would be of little interest. However, as the following theorem shows it is a useful intermediary for computing inertia groups of fibre bundles.

THEOREM 1. Let $M^{n} \rightarrow C \rightarrow N^{i}$ be a smooth fibre bundle with fibre $M^{n}$ and base manifold $N^{i}$. Then $\tilde{I}\left(M \times S^{i}\right) \subset I(C)$.

Proof. Let $G:\left(M \times S^{i}\right) \# \Sigma \rightarrow M \times S^{i}$ be a diffeomorphism satisfying $G \mid M \times\{*\}=$ identity, where $* \in D_{+}^{i}, \# \Sigma$ is performed in $M \times D_{-}^{i}$, and $S^{i}=$ $D_{+}^{i} \cup D_{-}^{i}$. By the tubular neighborhood and isotopy extension theorems, $G$ can be isotoped to $G^{\prime}$ satisfying $G^{\prime}\left(M \times D_{+}^{i}\right)=M \times D_{+}^{i}$. Let $G_{+}=G^{\prime} \mid M \times D_{+}^{i}$ and $G_{-}=G^{\prime} \mid\left(M \times D_{-}^{i}\right) \# \Sigma$, and observe that the composition $\tilde{G}=G_{+}^{-1}$ 。 $G_{-}:\left(M \times D^{i}\right) \# \Sigma \rightarrow M \times D^{i}$ restricts to the identity on the boundary. Take

Received by the editors May 29, 1981.

1980 Mathematics Subject Classification. Primary 57R22; Secondary 57R55.

Key words and phrases. Exotic sphere, inertia groups, fibre bundle, Whitehead group.

(C) 1982 American Mathematical Society 0002-9939/81/0000-1065/802.00 
$D^{i} \subset N$ and observe that since $C$ is trivial over $D^{i}, C$ admits a decomposition $C=\left(M \times D^{i}\right) \cup\left(C \backslash\left(M \times \operatorname{int} D^{i}\right)\right)$.

The diffeomorphism defined by the following diagram

$$
\begin{array}{rlrl}
\Sigma \# C & =\left(\left(M \times D^{i}\right) \# \Sigma\right) & \cup & \left(C \backslash\left(M \times \operatorname{int} D^{i}\right)\right) \\
\tilde{G} \downarrow & & \downarrow \text { identity } \\
C & =\left(M \times D^{i}\right) & \cup & \left(C \backslash\left(M \times \operatorname{int} D^{i}\right)\right)
\end{array}
$$

shows $\Sigma \in I(C)$. This completes the proof.

Examples of nontrivial elements in $\tilde{I}\left(M \times S^{i}\right)$ can be constructed easily for manifolds of the form $M^{n}=N^{n-j} \times S^{j}, j \geq i+2$. Take a generator $\alpha \in$ $\pi_{i}(S O(i+1))$ and let $s: S O(m) \rightarrow S O(m+1)$ denote the suspension map. Define a self-diffeomorphism $h$ of $N^{n-j} \times S^{j} \times S^{i}$ by $h(x, y, z)=\left(x, s_{*} \alpha(z) \cdot y, z\right)$, select $D^{j} \subset S^{j}$ satisfying $\left(s_{*} \alpha(z)\right) D^{j}=D^{j}$ for all $z \in S^{i}$, and observe that for $D^{n}=D^{n-j} \times D^{j} \subset N^{n-j} \times S^{j}, h \mid D^{n} \times S^{i}$ has the form $h((x, y), z)=$ $\left(x, s_{*} \alpha(z) \cdot y, z\right)=\left(s_{*}^{n-j+1} \alpha(z) \cdot(x, y), z\right)$. For some point $z_{0} \in S^{i}, s_{*} \alpha\left(z_{0}\right)=$ [identity] and $h \mid M \times\left\{z_{0}\right\}=$ identity. Suppose $\Sigma(f) \in I(M)$ so there is a diffeomorphism $\bar{f}: M^{n} \backslash \operatorname{int} D^{n} \rightarrow M^{n} \backslash \operatorname{int} D^{n}$ with $\bar{f} \mid \partial D^{n}=f$. Letting $h_{0}$ denote $h \mid\left(M \backslash \operatorname{int} D^{n}\right) \times S^{i}$, the commutator $\left(\bar{f}^{-1} \times\right.$ id $) h_{0}^{-1}(\bar{f} \times$ id $) h_{0}$ restricted to the boundary represents the element $\tau_{n-1, i}\left(f, s_{*}^{n-j+1}(\alpha)\right)=\beta$ in the image of the Milnor-Munkres-Novikov pairing $\tau_{n-1, i}: \Gamma^{n} \otimes \pi_{i}(S O(n)) \rightarrow \Gamma^{n+i}$ (see p. 834 of [10]). Lemma 1 of [7] shows there is a diffeomorphism $F:\left(M \times S^{i}\right) \# \Sigma(\beta) \rightarrow$ $M \times S^{i}$ and it is easy to see this diffeomorphism satisfies $F \mid M \times\left\{z_{0}\right\}=$ identity. This proves

Corollary. Let $M^{n}=N^{n-j} \times S^{j}, j \geq i+2$. Then for any $\Sigma(f) \in I(M)$, $\Sigma(\beta)$ belongs to $\tilde{I}\left(M \times S^{i}\right)$ and hence to $I(C)$ for each fibre bundle $M^{n} \rightarrow C \rightarrow N^{i}$, where $\beta=\tau_{n-1, i}\left(f, s_{*}^{n-j+1}(\alpha)\right)$, $\alpha$ a generator of $\pi_{i}(S O(i+1))$.

3. Some calculations of $\tilde{I}\left(M \times S^{i}\right)$. To apply Theorem 1 some specific calculations of $\tilde{I}\left(M \times S^{i}\right)$ are needed. Theorems 2 and 3 provide examples.

THEOREM 2. If $M^{n}$ is compact, $n \geq 5, \pi_{1} M$ a torsion group, and $\mathrm{Wh}\left(\pi_{1} M\right)=$ 0 , then $I\left(M \times S^{1}\right)=\tilde{I}\left(M \times S^{1}\right)$.

Proof. Let $G:\left(M \times S^{1}\right) \# \Sigma \rightarrow M \times S^{1}$ be a diffeomorphism and observe that since $\pi_{1} M$ is a torsion group, $G_{\#}\left(\pi_{1} M\right)=\pi_{1} M$ and so $G$ induces a diffeomorphism $\hat{G}:(M \times \mathbf{R}) \# \mathbf{Z} \Sigma \rightarrow M \times \mathbf{R}$ of the infinite cyclic coverings. Since $M$ is compact, $\hat{G}(M \times 0)$ lies above some $M \times t \subset M \times \mathbf{R}$ and the regions $A$ between $\hat{G}(M \times 0)$ and $M \times t$ and $B$ between $\hat{G}(M \times 1)$ and $M \times(t+1)$ are $h$-cobordisms. By the $s$-cobordism theorem the vanishing of $\mathrm{Wh}\left(\pi_{1} M\right)$ implies there are trivializations $\tau: A \rightarrow M \times[t, 0]$ and $T \tau T^{-1}: B \rightarrow M \times[t+1,1]$, where $T:(x, t) \rightarrow(x, t+1)$ is the covering translation corresponding to the projection $M \times \mathbf{R} \rightarrow M \times S^{1}$. Then $\left(T \tau T^{-1} \mid B \backslash A\right) \circ(\hat{G} \mid(M \times[0,1]) \# \Sigma)$ projects to a diffeomorphism $H:(M \times$ $\left.S^{1}\right) \# \Sigma \rightarrow M \times S^{1}$ satisfying $H(M \times\{*\})=M \times\{*\}$, hence $\Sigma \in \tilde{I}\left(M \times S^{1}\right)$. This completes the proof.

Denoting by $M_{g}$ the mapping torus $M \times[0,1] /\{(m, 0) \sim(g(m), 1)\}$ where $g \in$ $\operatorname{Diff}(M)$, it follows from Theorems 1 and 2 that $I\left(M \times S^{1}\right) \subset I\left(M_{g}\right)$ for all $g \in$ $\operatorname{Diff}(M)$. Examples of manifolds $M$ satisfying the hypotheses of Theorem 2 and having $I\left(M \times S^{1}\right) \neq 0$ can be constructed using Proposition 1 and Theorem 2 of 
[12] and also using Theorem 1 of [7]. For another example observe that Theorem 2 implies $I\left(\mathbf{C} P(3) \times S^{1}\right)=\tilde{I}\left(\mathbf{C} P(3) \times S^{1}\right)$, and by $I .10$ of $[3] I\left(\mathbf{C P}(3) \times S^{1}\right)=$ $\mathbf{z}_{7} \subset \mathbf{Z}_{28}=\theta_{7}$. Thus for any mapping torus $M$ with fibre $\mathbf{C}(3), \mathbf{z}_{7} \subset I(M)$. Note that by Corollary 2 of [11] there are nontrivial mapping tori with fibre $\mathbf{C P}(3)$ since $\pi_{0} \operatorname{Diff}(\mathbf{C P}(3)) \neq 0$ by $\Pi 1.11$ of $[3]$.

An argument from [11] can be combined with Theorem 2 to yield

COROLlARY. If $M^{n}$ is compact, $n \geq 5, \pi_{1} M$ a torsion group, and $\mathrm{Wh}\left(\pi_{1} M\right)=$ 0 , then $I\left(M \times S^{1}\right)=I\left(M_{g}\right)$ for $g \in \operatorname{Diff}(M)$.

Proof. Theorems 1 and 2 imply $I\left(M \times S^{1}\right) \subset I\left(M_{g}\right)$. For the reverse inclusion, suppose $G: \Sigma \# M_{g} \rightarrow M_{g}$ is a diffeomorphism. Let $f \in \operatorname{Diff}\left(D^{n}, \operatorname{rel} \partial D^{n}\right)$ represent $\Sigma$ and define a diffeomorphism $\psi(f) \in \operatorname{Diff}(M)$ by $\psi(f) \mid D^{n}=f$ and $\psi(f) \mid M \backslash$ int $D^{n}=$ identity, for some $D^{n} \subset M$. By Lemma 1 of [2] there is a diffeomorphism $H: M_{g \psi(f)} \rightarrow M_{g} \# \Sigma$, and by Corollary 4 of [11] the diffeomorphism $G H: M_{g \psi(f)} \rightarrow M_{g}$ is pseudo-isotopic to $F: M_{g \psi(f)} \rightarrow M_{g}$ with $F \mid M=$ identity. Then $F H^{-1}: M_{g} \# \Sigma \rightarrow M_{g}$ satisfies $F H^{-1} \mid M=$ identity, so splitting $M_{g} \# \Sigma$ and $M_{g}$ along $M$ and reglueing by the identity gives a diffeomorphism $\left(M \times S^{1}\right) \# \Sigma \rightarrow M \times S^{1}$. This completes the proof.

In order to apply Theorem 2 and its corollary, some information is needed about which finite groups $\pi$ have $\mathrm{Wh}(\pi)=0$. If $\pi$ is finite abelian, $\mathrm{Wh}(\pi)=S K_{1}(\mathrm{Z} \pi) \oplus$ $U(\mathbf{Z} \pi)$ (p. 202 of [16]). For finite cyclic groups $\mathbf{Z}_{q}$ it follows from a Theorem of Bass (XI.7.3 of [1]) that $S K_{1}\left(\mathbf{Z Z}_{q}\right)=0$, and work of Bass, Higman, Milnor, and Serre (11.5 of [4]) shows $\mathrm{Wh}\left(\mathrm{Z}_{q}\right)=0$ if and only if $q=1,2,3,4$, or 6. Another Theorem of Bass (6.4 of [14]) shows that $U(\mathrm{Z} \pi)=0$ if $\pi$ is finite abelian of exponent 1 , $2,3,4$, or 6. Combining this with calculations of Stein (pp. 206 and 204 of [16]) shows $\mathrm{Wh}\left(\mathbf{Z}_{3}^{2}\right)=0$ and $\mathrm{Wh}\left(\mathbf{Z}_{2}^{k}\right)=0$ for all $k$. For nonabelian examples, Milnor applies Bass' theorem (pp. 374 of [14]) to show for the symmetric group $S_{n}$ that $\mathrm{Wh}\left(S_{n}\right)$ is finite, and Magurn (7.3 of [16]) shows $S K_{1}\left(S_{n}\right)=0$ for $n=4,5$, or 6, so $\mathrm{Wh}\left(S_{n}\right)=0$ for $n=4,5$, or 6, by a result of Wall (pp. 209-210 of [16]). Finally, Magurn has shown (p. 210 of [16]) that $S K_{1}\left(\mathrm{Z} D_{k}\right)=0$ for any finite dihedral group $D_{k}$, and an easy calculation using 39.5 and 47.15 of [5] shows $\mathrm{Wh}\left(D_{k}\right)=0$ for $k=3,4$, and 6 .

For another calculation of $\tilde{I}\left(M \times S^{i}\right)$, recall Brumfiel's definition [3, Appendix II] of the special inertia group $I_{h}(M)=\left\{\Sigma \in \theta_{n}\right.$ : there is a diffeomorphism $M \rightarrow M \# \Sigma$ homotopic to $\iota\}$, where the PL homeomorphism

$$
\iota: M=\left(M \backslash \operatorname{int} D^{n}\right) \cup_{\text {id }} D^{n} \rightarrow\left(M \backslash \operatorname{int} D^{n}\right) \cup_{f} D^{n}=M \# \Sigma
$$

is defined by $\iota \mid M \backslash \operatorname{int} D^{n}=$ identity and $\iota \mid D^{n}=c(f)=$ cone map on $f$.

THEOREM 3. If $n+2<i$, then $I_{h}\left(M \times S^{i}\right) \subset \tilde{I}\left(M \times S^{i}\right)$ and so $I_{h}\left(M \times S^{i}\right) \subset$ $I(C)$ for any fibre bundle $M^{n} \rightarrow C \rightarrow S^{i}$.

Proof. By hypothesis there is a diffeomorphism $G:\left(M \times S^{i}\right) \# \Sigma \rightarrow M \times S^{i}$ and a homotopy $F_{t}$ between $F_{0}=\iota$ and $F_{1}=G$. Since $n+2<i, F \mid(M \times\{*\} \times I)$ can be smoothed and self-general positioned to an imbedding giving rise to a pseudoisotopy $\tilde{F}: M \times\{*\} \times I \rightarrow M \times S^{i} \times I$. By Theorem 2.1 of [8] and the isotopy extension theorem, there is an isotopy $H: M \times S^{i} \times I \rightarrow M \times S^{i} \times I$ with $H_{0}=$ identity and $H_{1} \mid M=\tilde{F}_{1}$. Define $\bar{H}: M \times S^{i} \rightarrow M \times S^{i}$ by $\bar{H}(m, s)=H_{1}^{-1}(m, s)$. 
Then $\bar{H} G:\left(M \times S^{i}\right) \# \Sigma \rightarrow M \times S^{i}$ satisfies $\bar{H} G \mid M \times\{*\}=$ inclusion. This completes the proof.

As a final example, consider a diffeomorphism $f: \Sigma \#\left(M^{n} \times S^{i}\right) \rightarrow M^{n} \times S^{i}$. If $M$ is $(n-i+1)$-connected and $2 i-3 \geq n$, then $f$ is isotopic to $f^{\prime}$ with $f^{\prime} \mid M \times\{*\}=$ inclusion for some $* \in S^{i}$ if and only if a single obstruction

$$
\alpha\left(f(M \times\{*\}), M \times\{*\}: M \times S^{i}\right) \in \pi_{n-i}
$$

vanishes [19]. In particular, if $M^{n}$ is $(n-i+1)$-connected and $2 i-3 \geq n$, then $I\left(M \times S^{i}\right)=\tilde{I}\left(M \times S^{i}\right)$ if $n-i=4,5$, or 12 (since then $\pi_{n-i}$ vanishes [18]).

\section{REFERENCES}

1. H. Bass, Algebraic $K$-theory, Benjamin, New York, 1968.

2. W. Browder, Diffeomorphisms of 1-connected manifolds, Trans. Amer. Math. Soc. 128 (1967), 155-163.

3. G. Brumfiel, Homotopy equivalences of almost smooth manifolds, Comment Math. Helv. 46 (1971), 381-407.

4. M. Cohen, $A$ course in simple-homotopy theory, Graduate Texts in Mathematics, vol. 10, Springer-Verlag, New York, 1973.

5. C. Curtis and I. Reiner, Representation theory of finite groups and associate algebras, Wiley, New York, 1962.

6. R. DeSapio, Differentiable structures on a product of spheres. II, Ann. of Math. (2) 89 (1969), 305-313.

7. M. Frame, On the inertia groups of mapping tori and of products with spheres, Indiana Univ. Math. J. 29 (1980), 151-159.

8. J. Hudson, Concordance, isotopy, and diffeotopy, Ann. of Math. (2) 91 (1970), 425-448.

9. A. Kosinski, Inertia groups of $\pi$-manifolds, Amer. J. Math. 89 (1967), 227-248.

10. T. Lawson, Remarks on the pairings of Bredon, Milnor, and Milnor-Munkres-Novikov, Indiana Univ. Math. J. 22 (1973), 833-843.

11. __ Splitting isomorphisms of mapping tori, Trans. Amer. Math. Soc. 205 (1975), 285-294.

12. J. Levine, Inertia groups of manifolds and diffeomorphisms of spheres, Amer. J. Math. 92 (1970), 243-258.

13. J. Milnor, On manifolds homeomorphic to the 7-sphere, Ann. of Math. (2) 64 (1956), 399-405.

14. —, Whitehead torsion, Bull. Amer. Math. Soc. 72 (1966), 358-426.

15. R. Schultz, Smooth structures on $S^{p} \times S^{q}$, Ann. of Math. (2) 90 (1969), 187-198.

16. M. Stein, Whitehead groups of finite groups, Bull. Amer. Math. Soc. 84 (1978), 201-212.

17. I. Tamura, Sur les sommes connexes de certaines varió etó es diffó erentiables, C. R. Acad. Sci. Paris 255 (1962), 3104-3106.

18. H. Toda, Composition methods in homotopy groups of spheres, Ann. of Math. Studies, no. 49, Princeton Univ. Press, Princeton, N. J., 1962.

19. R. Wells, Modifying intersections, Illinois J. Math. 11 (1967), 389-403.

DEPARTMENT OF MATHEMATICS, UNIVERSITY OF WISCONSIN-PARKSIDE, KENOSHA, WISCONSIN 53141 